\title{
Analysis of Eddy Current Distribution in High Frequency Coaxial Transformer With Faraday Shield
}

\author{
Junwei $\mathrm{Lu}^{1}$ and Francis Dawson ${ }^{2}$ \\ ${ }^{1}$ Griffith School of Engineering, Faculty of Engineering and Information Technology, \\ Nathan Campus, Griffith University, Nathan, Brisbane, Qld 4111, Australia \\ ${ }^{2}$ Department of Electrical and Computer Engineering, University of Toronto, Toronto, ON M5S-3G4, Canada,
}

\begin{abstract}
This paper presents analysis and measurement results of a high-frequency (HF) coaxial transformer with Faraday shield. The simulation results demonstrate that the magnetic flux and eddy current distribution are not impacted by the inclusion of a Faraday shield. The Faraday shield's maximum eddy current density is less than $17 \%$ of the maximum density found in either of the other two windings. The experimental results demonstrate that the voltage ratio, coupling coefficient and load characteristic of the HF coaxial transformer with Faraday shield at frequencies up to $1 \mathrm{MHz}$ are in agreement with the turns ratio and load regulation for a transformer in which capacitive effects are neglected.
\end{abstract}

Index Terms-Coaxial transformer, eddy current, Faraday shield, high-frequency transformer.

\section{INTRODUCTION}

$\mathbf{E}$ LECTRICAL isolation of switching mode power supplies can be facilitated by employing high-frequency (HF) transformers in the design. The input power source and the output of the power supply are coupled together by the magnetic field. Practical transformers are not ideal and have leakage inductances and a capacitance between the primary and secondary windings. The capacitance couples HF noise from the primary winding to the secondary winding. The coupling noise can cause serious common mode problems and, thus, the design may not be electromagnetically compatible with the surrounding environment. The effects of the intra and inter winding capacitances cannot be ignored if the operating frequencies are above $100 \mathrm{kHz}$ or if the turns ratio is very large [1].

A Faraday shield, which is formed by placing a grounded conductor between the two windings, can eliminate the HF noise coupling. Ideally the shield should not influence the magnetic coupling, but should eliminate the capacitive coupling between windings when the shield is grounded. The shielding coil may exhibit, at higher frequencies, an eddy-current loss caused by leakage flux. The location and thickness of the shield should be designed in such a way as to minimize the impact of eddy current loss and insertion loss which is caused by coupling capacitance between the primary and secondary windings.

This main purpose of this paper is to investigate the eddy current loss of the Faraday shield and the coupling coefficient of the HF coaxial transformer with Faraday shield placed between the primary and secondary windings.

\section{CoAXIAL Transformer With FARAdAy ShIELD}

The basic structure of the coaxial transformer [2], with Faraday shield, is shown in Fig. 1. The outer copper tube is used as a primary winding, the wires inside are used as a

Digital Object Identifier 10.1109/TMAG.2006.880093

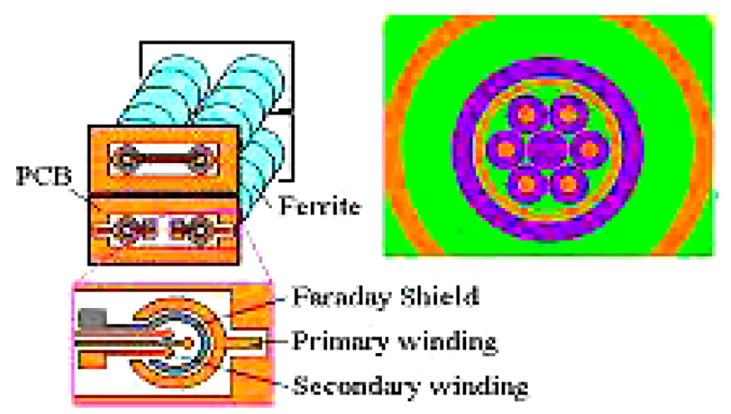

Fig. 1. Coaxial Structure with a Faraday shield (E field shielding). (Color version available online at http://ieeexplore.ieee.org.)

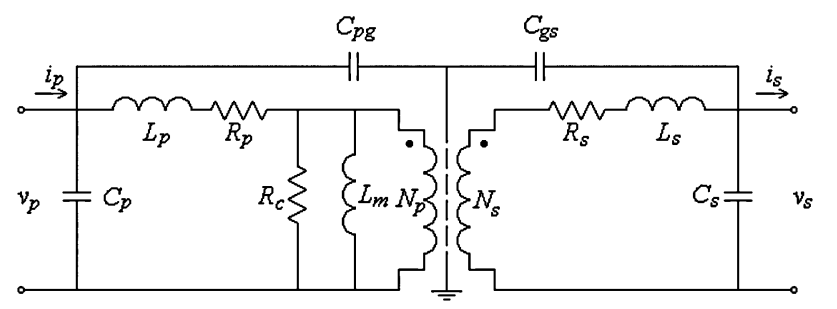

Fig. 2. HF equivalent circuits of transformers with Faraday shield.

secondary winding (two wires in parallel connection to form a three turns' winding) and the copper tube in between the primary winding (as one turn winding) and the secondary winding represents the Faraday shield.

Fig. 2 shows the HF equivalent circuit of a HF transformer with one Faraday shield. The coupling capacitance between the primary and secondary windings can be reduced by the grounded Faraday shield.

A three-shield structure is used if noise coupling between the primary and secondary windings of a single shield device is too high. In practice, the residual parasitic capacitance between windings cannot be totally eliminated, but this capacitance is too small to be significant up to approximately $10 \mathrm{MHz}$. After applying shielded transformers in power electronic equipments, a 20-40 dB EMI reduction can be expected compared to conventional transformer [1]. Moreover, the shielded transformer 
also offers some protection to semiconductors against transients propagating from the utility side.

At higher power levels and higher frequencies it is important to reduce the magnetic leakage fields and eddy current loss in the windings. A coaxial transformer with windings placed inside ferrite cores is best suited for this purpose as it has a lower eddy current loss [3]. Moreover, the Faraday shield with such a coaxial structure placed between primary and secondary windings also exhibits a higher coupling coefficient compared to a conventional HF transformer structure, including a planar transformer.

With increasing frequency, the effect of leakage inductances and parasitic capacitances grow and the insertion loss cannot be neglected. The correct description of the insertion loss at higher frequencies is very difficult to obtain and needs to incorporate additional modeling approximations. The reason for this is that the turns-ratio of the transformer can no longer be calculated as the ratio of the turns, because the flux of the ferrite core is decreased by eddy current and hysteresis losses. The insertion loss of the transformer is determined by the primary leakage inductance, $L_{\mathrm{p}}$ and secondary leakage inductance, $L_{\mathrm{s}}$ and winding losses in the middle HF range $(>100 \mathrm{KHz})$. The turns-ratio of the transformer can be approximated by

$$
r=\sqrt{L_{p} / L_{s}}
$$

where $L_{\mathrm{p}}$ is the primary leakage inductance and $L_{\mathrm{s}}$ the secondary leakage inductance [1].

With a further increase in frequency $(>1 \mathrm{MHz})$, the capacitive coupling between the windings continues to grow. Under these conditions, the insertion loss is determined predominantly by the intra-capacitance of the windings and the capacitive coupling between the windings. The turns-ratio of the transformer can be expressed approximately as

$$
r=\left(1+C_{s} / C_{\mathrm{ps}}\right)
$$

where $C_{\mathrm{s}}$ and $C_{\mathrm{ps}}$ are secondary parasitic capacitance and coupling capacitance, respectively [1].

\section{Computation Results of CoAXial TRAnsformer}

A BEM-based numerical simulator, 2D Time Harmonic Eddy Current Simulator, Oersted $\odot$ [4] is used to investigate the effectiveness of a Faraday shield at high frequencies. The shielding between the primary and secondary windings is usually constructed of a one-layer winding or of an isolating sheet covered on one side by a conducting layer. In this coaxial transformer, the shielding layer is made of a single layer of thin copper tube which has the same structural configuration as the primary winding. The magnetic flux distribution within the HF transformer with and without a Faraday shield is simulated at an operating frequency of $1 \mathrm{MHz}$. The simulation results in Fig. 3(a) and (b) show that the flux distributions around the winding structure of the transformer have a very similar pattern as a design without a Faraday shield, which implies that Faraday shield, placed between the primary winding and the secondary

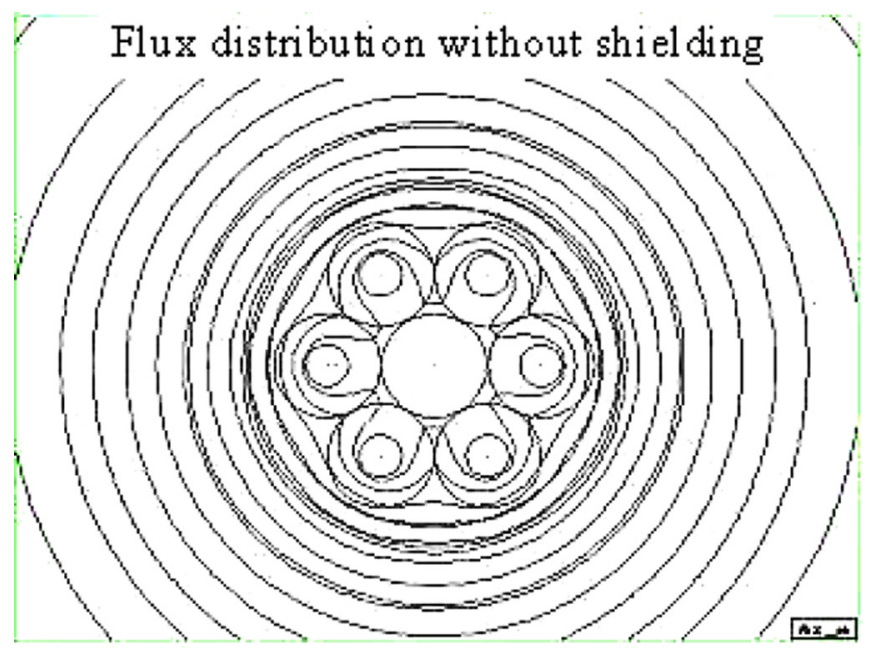

(a)

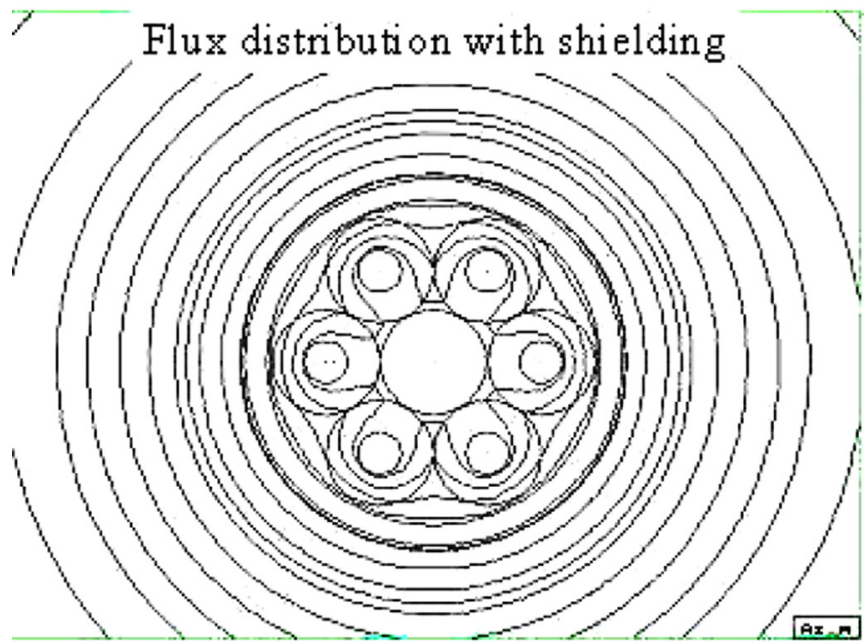

(b)

Fig. 3. Magnetic flux distribution of the HF coaxial transformer (a) without Faraday shield and (b) with Faraday shield at an operating frequency of $1 \mathrm{MHz}$.

winding of the coaxial transformer, does not influence the magnetic flux coupling and distribution.

Fig. 4(a) and (b) shows the eddy-current distributions in the windings without and with a shielding coil, where the excitation source is applied to the primary winding. The numerical simulation results indicate that the eddy-current density in the shielding coil is relatively small compared with the eddycurrent in the secondary windings. The maximum eddy current density in the Faraday shield is $<5 \mathrm{~A} / \mathrm{cm}^{2}$, while the maximum eddy current density in the secondary windings is 30.07 $\mathrm{A} / \mathrm{m}^{2}$ (without shielding coil) and $30.40 \mathrm{~A} / \mathrm{m}^{2}$ (with shielding coil), respectively,. The maximum eddy current density in the Faraday shield was found to be less than $17 \%$ compared with the one in the secondary windings. Therefore, the power loss in the Faraday shield can be neglected. Both eddy current and magnetic flux distributions in the transformer windings with the Faraday shield remains the same as the current distribution without the Faraday shield, which indicates that the Faraday shield does not have impact on transformer operation at HF. 


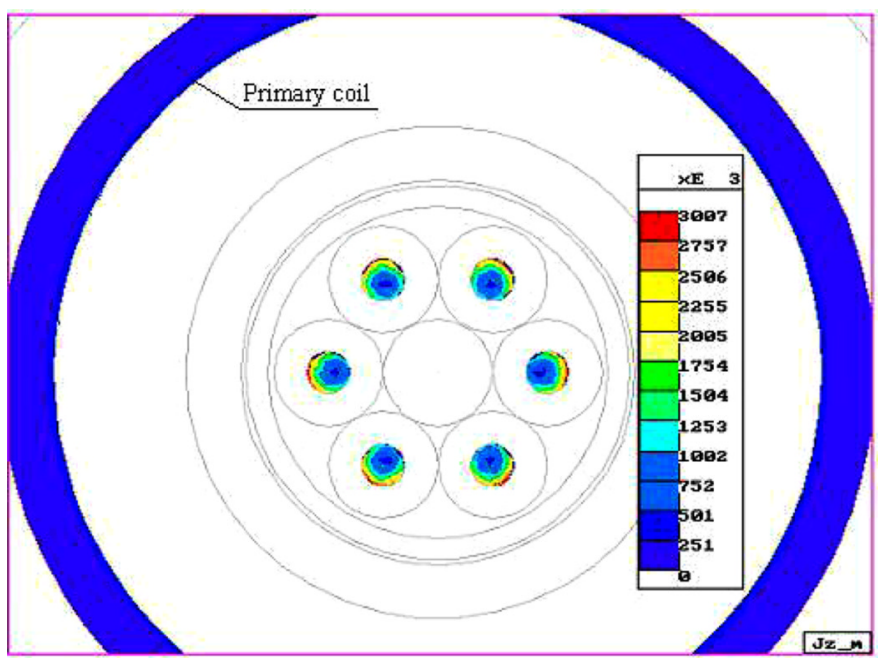

(a)

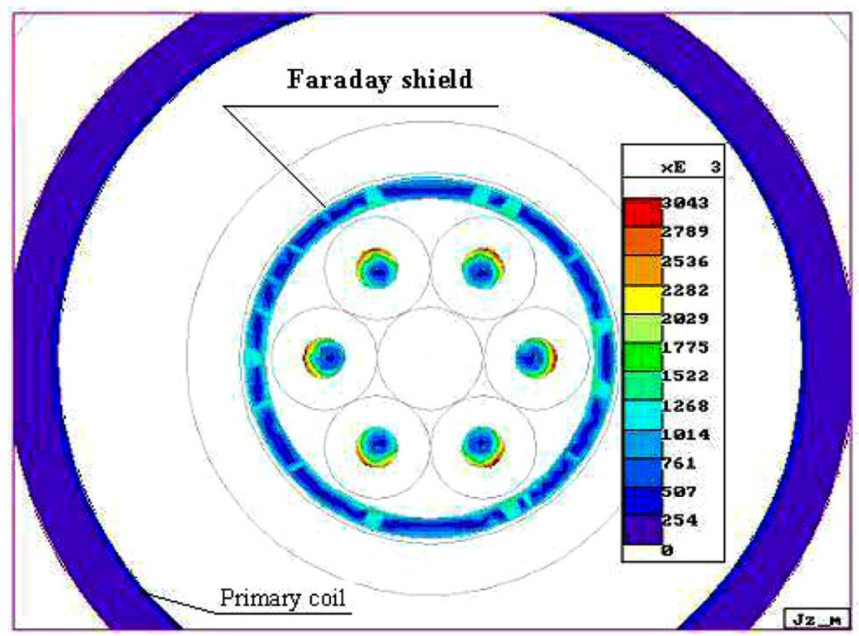

(b)

Fig. 4. Eddy current distribution of the HF coaxial transformer (a) without Faraday shield and (b) with Faraday shield at an operating frequency of $1 \mathrm{MHz}$. (Color version available online at http://ieeexplore.ieee.org.)

\section{EXPERIMENTAL RESULTS FOR COAXIAL TRANSFORMER}

The transformer with Faraday shield has been tested with a load (100 $\Omega$ ) using a single switch forward resonant converter configuration. A $1.144 \mathrm{MHz}$ switching frequency is used to investigate the characteristic of the transformer. The measured waveforms for an operating frequency of $1.144 \mathrm{MHz}$ are illustrated in Fig. 5 [5], where the waveform (Channel 2) at the top of Fig. 5 is measured between the gate and the source of the switching MOSFET. The current flowing through the primary winding is shown on channel 1 . Channel 3 is the drain to source waveform $\left(\mathrm{V}_{\mathrm{DS}}\right)$ of the MOSFET. The output voltage from the secondary winding of the prototype HF transformer is monitored on channel 4 using a Tektronix 4-channel Digitising Oscilloscope. The peak to peak voltage ratio was measured as $1: 3.62$ for an operating frequency of $1.144 \mathrm{MHz}$. The low frequency turns-ratio of the transformer is $1: 3$ which quite obviously is not in agreement with the measured turns ratio. It is obvious that the ratio of the transformer is determined by leakage inductance ratio $r=\sqrt{L_{p} / L_{s}}$ at this frequency. The secondary leakage in-

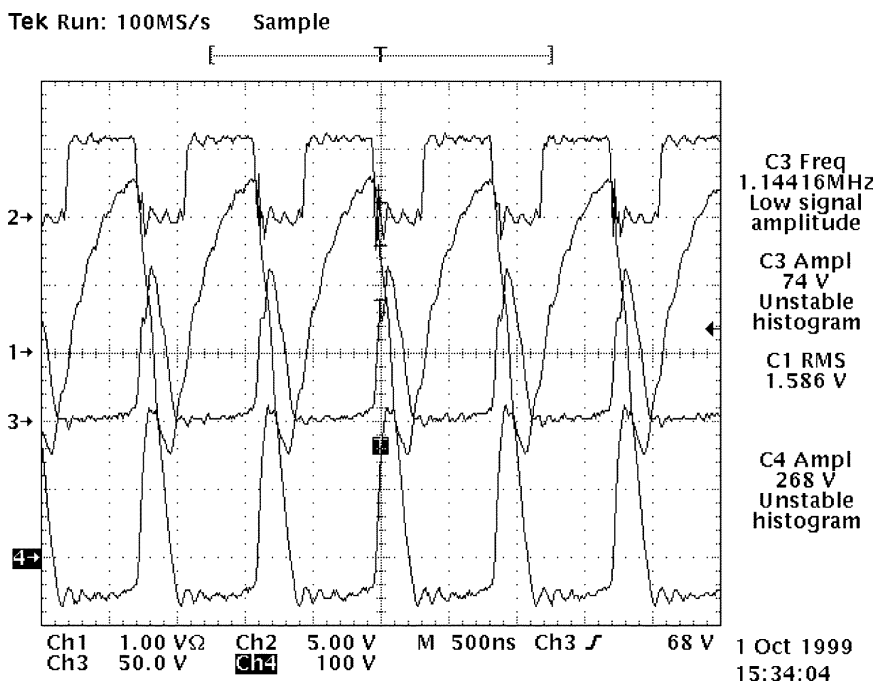

Fig. 5. Waveforms versus time (time scale is $500 \mathrm{~ns} / \mathrm{per}$ block indicated at the second bottom line of waveform plot) for a transformer with single Faraday shield at operating frequency of $1.144 \mathrm{MHz}$.

ductance is much larger than the primary one due to the extra length of the tracks on the printed circuit board at both ends of the transformer.

\section{CONCLUSION}

An HF coaxial transformer with shielding coil has been constructed and tested. The numerical and experimental results show that the Faraday shield had no effect on the magnetic coupling coefficient, and the flux and eddy current distributions in the coaxial HF transformer. The maximum eddy current density in the Faraday shield was found to be less than $17 \%$ compared with the one in secondary windings. The eddy current in the secondary winding is higher than in the primary winding. This shielded coaxial transformer is suitable for HF operation, because it has negligible capacitive coupling between the primary and secondary windings and low insertion loss under HF operating conditions. Moreover, the HF transformer with shielding has provided the best EMI suppression effect compared to conventional HF transformer with a $20-40 \mathrm{~dB}$ reduction for differential-mode EMI.

\section{REFERENCES}

[1] L. Tihanyi, Electromagnetic Compatibility in Power Electronics. Piscataway, NY: IEEE, 1995.

[2] J. Lu, F. P. Dawson, and S. Yamada, "Application and analysis of adjustable profile high frequency switch mode transformer having a U-shaped winding structure," IEEE Trans. Magn., vol. 34, no. 4, pp. 1345-1347, Jul. 1998.

[3] K. W. Klontz et al., "An active cooled $120 \mathrm{~kW}$ coaxial winding transformer for fast charging electric vehicles," IEEE Trans. Ind. Appl., vol. 31, no. 6, pp. 1257-1263, Nov./Dec. 1995.

[4] 2D Time Harmonic Eddy Current Simulator, 1999. Integrated Engineering Software, Inc., IES Oersted $\odot$.

[5] F. Wong et al., "Effectiveness of shielded high frequency coaxial transformer for switching power supplies," in Proc. EMC2002, vol. 5, 2002.

Manuscript received March 3, 2006; revised June 10, 2006 (e-mail: j.lu@ griffith.edu.au). 